\title{
Space Shuttle Underside Astronaut Communications Performance Evaluation
}

\author{
Shian U. Hwu, Justin A. Dobbins, Yin-Chung Loh, Quin D. Kroll, and Catherine C. Sham
}

\begin{abstract}
The Space Shuttle Ultra High Frequency (UHF) communications system is planned to provide Radio Frequency (RF) coverage for astronauts working underside of the Space Shuttle Orbiter (SSO) for thermal tile inspection and repairing. This study is to assess the Space Shuttle UHF communication performance for astronauts in the shadow region without line-of-sight (LOS) to the Space Shuttle and Space Station UHF antennas. To insure the RF coverage performance at anticipated astronaut worksites, the link margin between the UHF antennas and Extravehicular Activity (EVA) Astronauts with significant vehicle structure blockage was analyzed. A series of near-field measurements were performed using the NASA/JSC Anechoic Chamber Antenna test facilities. Computational investigations were also performed using the electromagnetic modeling techniques. The computer simulation tool based on the Geometrical Theory of Diffraction (GTD) was used to compute the signal strengths. The signal strength was obtained by computing the reflected and diffracted fields along the propagation paths between the transmitting and receiving antennas. Based on the results obtained in this study, RF coverage for UHF communication links was determined for the anticipated astronaut worksite in the shadow region underneath the Space Shuttle.
\end{abstract}




\title{
Space Shuttle Underside Astronaut Communications Performance Evaluation
}

\author{
Shian U. Hwu, Justin A. Dobbins, Yin-Chung Loh, Quin D. Kroll, and Catherine C. Sham
}

\begin{abstract}
The Space Shuttle Ultra High Frequency (UHF) communications system is planned to provide Radio Frequency (RF) coverage for astronauts working underside of the Space Shuttle Orbiter (SSO) for thermal tile inspection and repairing. This study is to assess the Space Shuttle UHF communication performance for astronauts in the shadow region without line-of-sight (LOS) to the Space Shuttle and Space Station UHF antennas. To insure the RF coverage performance at anticipated astronaut worksites, the link margin between the UHF antennas and Extravehicular Activity (EVA) Astronauts with significant vehicle structure blockage was analyzed. A series of near-field measurements were performed using the NASA/JSC Anechoic Chamber Antenna test facilities. Computational investigations were also performed using the electromagnetic modeling techniques. The computer simulation tool based on the Geometrical Theory of Diffraction (GTD) was used to compute the signal strengths. The signal strength was obtained by computing the reflected and diffracted fields along the propagation paths between the transmitting and receiving antennas. Based on the results obtained in this study, RF coverage for UHF communication links was determined for the anticipated astronaut worksite in the shadow region underneath the Space Shuttle.
\end{abstract}

Index Terms-Geometrical theory of diffraction, Space shuttles, Space vehicle communication, UHF communication.

\section{INTRODUCTION}

$\mathrm{T}$ HE Space Shuttle Orbiter (SSO) payload bay Ultra High 1 Frequency (UHF) antenna is planned to provide UHF communication links for Extravehicular Activity (EVA) astronauts in the worksite underneath the Space Shuttle for thermal tile inspection and repairing, as shown in Figure 1. The payload bay antenna pattern will be blocked and distorted due to

Manuscript received October 14,2004. This work was supported in part by the NASA Johnson Space Center under Contract NAS9-19100.

Shian U. Hwu is with Lockheed Martin Space Operations, Houston, Texas 77058 USA (phone: 281-333-7559; fax: 281-333-7620; e-mail: shian.u.hwul@jsc.nasa.gov).

Justin A. Dobbins is with NASA Johnson Space Center, Houston, Texas 77058 USA. (e-mail: justin.a.dobbins1@jsc.nasa.gov).

Yin-Chung Loh is with Lockheed Martin Space Operations, Houston, Texas 77058 USA (e-mail: y.c.loh@Imco.com).

Quin D. Kroll is with NASA Johnson Space Center, Houston, Texas 77058 USA. (e-mail: quin.kroll-1@nasa.gov).

Catherine C. Sham is with NASA Johnson Space Center, Houston, Texas 77058 USA. (e-mail: catherine.c.sham@nasa.gov). the Space Shuttle structure obstructions. The Radio Frequency (RF) signals have to overcome the vehicle structure blockage along the propagation paths. To ensure the RF coverage can meet the requirements, the link margin evaluation must take into account such antenna pattern distortion and signal attenuation due to blockage. The system must be able to compensate for the signal attenuation, and provide adequate signal level throughout designated areas of coverage for the communication links to function effectively.

In shadow regions, such non-line-of-sight (NLOS) locations will experience relatively high path loss and signal degradation. The signal degradation and path loss caused by structure blockages are in general much higher for signals in the microwave frequency bands, such as Wireless LAN (2.4 and 5 $\mathrm{GHz}$ ), than for those used by the Space Shuttle astronaut communications at the UHF frequency bands (414.2 and 417.1 $\mathrm{MHz})$.

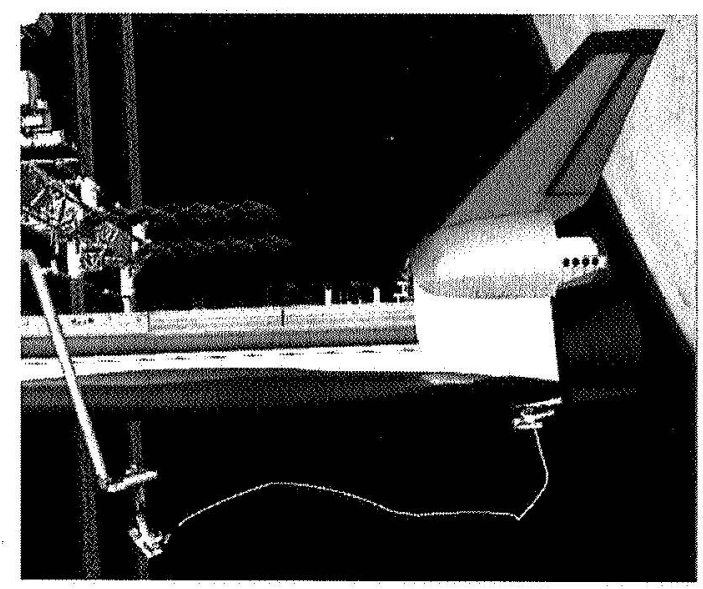

Fig. 1. Astronauts in worksite underneath Space Shuttle for thermal tile inspection and repairing.

A key goal of the work was to avoid the assumption that any blockages of the line-of-sight (LOS) automatically precluded the site from having communication coverage. Instead, This study is based on a detailed 3D electromagnetic modeling, which took into account diffractions along propagation paths over and around blockage structures. In many cases, as predicted by the computer simulations and supported by the experimental measurements, the diffractions can result in adequate received signals and link margin for communication coverage. 
The purpose of this study is to assess the signal strengths and RF coverage performance for astronauts working in the shadow region underneath the Space Shuttle. Both experimental measurements and computer simulations were performed. Due to the large size of the Space Shuttle, full-scale vehicle mockup measurements requiring a large outdoor range are difficult to implement. The scaled Space Shuttle mockup measurements in Anechoic Chamber are feasible. Another approach in evaluating the communication performance under vehicle blockage is using an electromagnetic modeling technique [1-7]. Computer simulations have advantages when (1) it is too expensive and dangerous to perform real tests or experiments on an existing complex vehicle; (2) the vehicle is not available or cannot be tested for a real scenario.

Using simulation tools for initial investigation before the actual measurements are performed for the final configuration can reduce the project costs and time. A reliable analytical tool provides matrix comparison for proposed approaches and helps to find the best solutions. Modern high-speed computers with large storage capacity have made possible the theoretical calculation of the signal strength with antennas mounted on complex structures such as Space Shuttle. The main advantage of this mathematical method is that, once the geometry of the vehicle has been represented in the computer, the analyses of different astronaut positions can be easily evaluated. The RF coverage analysis can be performed on the basis of a computer evaluation; the number of measurements can be cut down to a reasonable minimum for verification and validation purpose.

Since the Space Shuttle is large in terms of wavelength, the Geometrical Theory of Diffraction method (GTD) is a suitable candidate for the computational task. The Method of Moments (MoM) is a feasible tool that provides better accuracy. However, excessive computer resources and computing time will be required for electrically large Space Shuttle structure models. The MoM technique is suitable to verify GTD results with partial structure models immediate to the antenna. In this study, the GTD was used to compute the electric fields. The total field was obtained by summing the direct fields from the antennas and the reflected and diffracted fields along the propagation paths between the transmitting and receiving antennas. The computed signal strengths were compared to the signal strength corresponding to the $0 \mathrm{~dB}$ link margin. The RF coverage was determined for proposed astronaut worksites.

\section{COMPUTATIONAL METHOD}

The Geometrical Theory of Diffractions was used in the simulations to take into account the multipath and blockage effects from the Space Shuttle vehicle structures.

\section{A. Assumptions}

It is assumed that the electromagnetic properties of the Space Shuttle structures are such that the surfaces are highly reflective at the UHF frequency. Thus, the Space Shuttle structures are modeled as perfect electrical conductors.

The Space Shuttle payload bay and Space Station UHF antennas are quadrifilar helical antennas. The operating frequencies are $414.2 \mathrm{MHz}$ and $417.1 \mathrm{MHz}$. An average frequency of $415.65 \mathrm{MHz}$ was used in this analysis for the UHF communications.

\section{B. Signal Strength Computations}

At high frequencies the scattering fields depend on the electrical and geometrical properties of the scatterer in the immediate neighborhood of the point of reflection and diffraction. In the field computation, the incident, reflected, and diffracted fields are determined by the field incident on the reflection or diffraction point multiplied by a dyadic reflection or diffraction coefficient, a spreading factor, and a phase term. The reflected and diffracted field at a field point $\mathrm{r}^{\prime}, \boldsymbol{E}^{\mathrm{s}, \mathrm{d}}\left(\mathrm{r}^{\prime}\right)$, in general have the following form:

$$
E^{\mathrm{rdd}}\left(\mathrm{r}^{\prime}\right)=\boldsymbol{E}^{\mathrm{i}}(\mathrm{r}) \boldsymbol{D}^{\mathrm{r}, \mathrm{d}} A^{\mathrm{r}, \mathrm{d}}(\mathrm{s}) \mathrm{e}^{-\mathrm{jks}}
$$

where $E^{\mathrm{i}}(r)$ is the field incident on the reflection or diffraction point $r, D^{r, d}$ is a dyadic reflection or diffraction coefficient, $A^{\mathrm{r}, \mathrm{d}}(\mathrm{s})$ is a spreading factor, and $\mathrm{s}$ is the distance from the reflection or diffraction point $r$ to the field point r'. $D^{\mathrm{r}, \mathrm{d}}$ and $\boldsymbol{A}^{\mathrm{r}, \mathrm{d}}$ can be found from the geometry of the structure at reflection or diffraction point $r$ and the properties of the incident wave there. Thus, the total fields $\left(E^{\text {tot }}\right)$ can be obtained by summing up the individual contributions of the direct field $\left(\mathrm{E}^{\mathrm{di}}\right)$, reflected field $\left(\mathrm{E}^{\text {ref }}\right)$, and diffracted field ( $\left.\mathrm{E}^{\mathrm{dif}}\right)$ along the propagation paths, as following,

$$
E^{t o t}=E^{d i r}+\sum_{n=1}^{N} E_{n}^{r e f}+\sum_{m=1}^{M} E_{m}^{d i f}
$$

$\mathrm{E}^{\text {tot }}$ : Total field at the observation point,

$\mathrm{E}^{\mathrm{dir}}$ : Direct fields from antennas,

$\mathrm{E}^{\text {ref }}$ : Reflected fields from plates and curve surfaces,

$\mathrm{E}^{\mathrm{dif}}$ : Diffracted fields from plates and curve surfaces.

\section{EXPERIMENTAL INVESTIGATION}

The experimental data were used to verify the antenna performance and to assess the validity of the computer simulation. A series of the Space Shuttle UHF antenna near-field measurements based on the 1/10th scale Space Shuttle mockup were conducted in the NASA Johnson Space Center (JSC) Anechoic Chamber antenna test facility.

The Anechoic Chamber in the NASA Johnson Space Center has a 1/10th scale Orbiter mockup, as shown in Fig. 2, that was constructed for antenna testing. The $1 / 10$ th scale UHF antenna was mounted inside the payload bay in this Orbiter mockup. Since the entire test was performed with $1 / 10$ th scale hardware, the frequencies used for the testing were 10 times higher than 
the original frequency (e.g. $4.1565 \mathrm{GHz}$ to model the center frequency of $0.41565 \mathrm{GHz}$ ). The Space Shuttle near-field test setup is shown in Fig. 3. The signal strengths underneath the Space Shuttle were measured for the UHF antenna in the Space Shuttle payload bay.

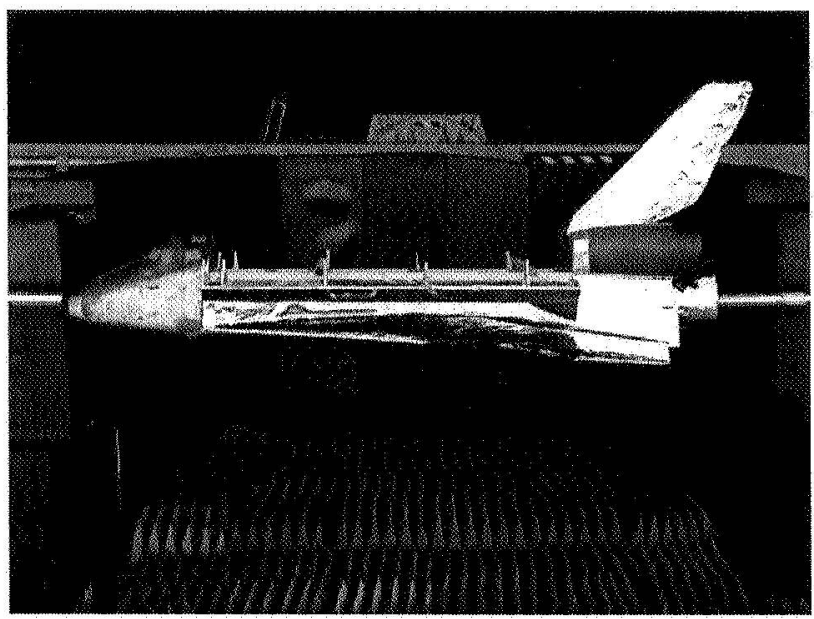

Fig. 2. The 1/10th scale SSO mockup in NASA/JSC Anechoic Chamber.

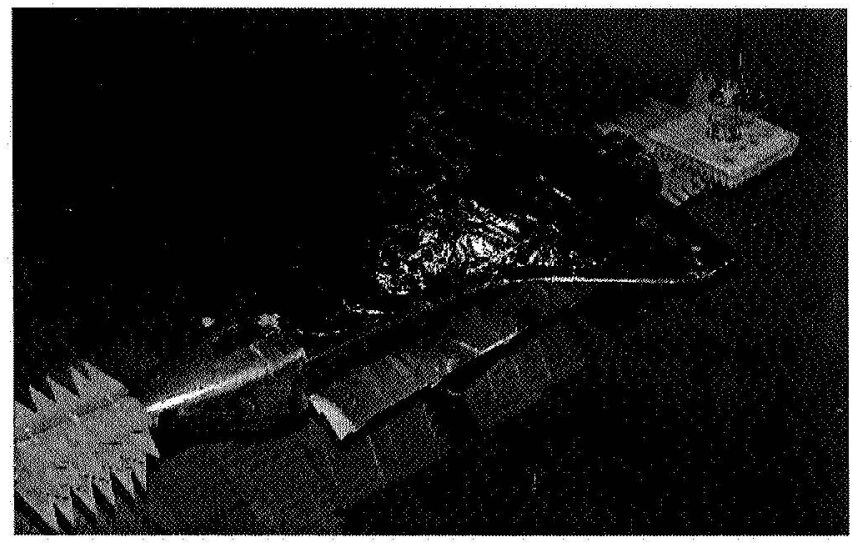

Fig. 3. Near-field measurement setup in NASAJJSC Anechoic Chamber.

\section{COMPUTER Simulation VAlidation}

The signal strengths along EVA paths underneath the Space Shuttle using the payload bay UHF antenna were computed using the GTD simulation tool. The computed electric fields were compared to the signal strength corresponding to the $0 \mathrm{~dB}$ link margin. Experimental data were obtained from the NASA/JSC Anechoic Chamber near-field measurements. The comparisons of measured and computed signal strength in the shadow region underneath a flat plate were shown in Figures 4 and 5. The comparisons of measured and computed signal strength in the shadow region underneath the Space Shuttle were shown in Figures 6 and 7. Reasonable agreement between measured and computed results is observed.

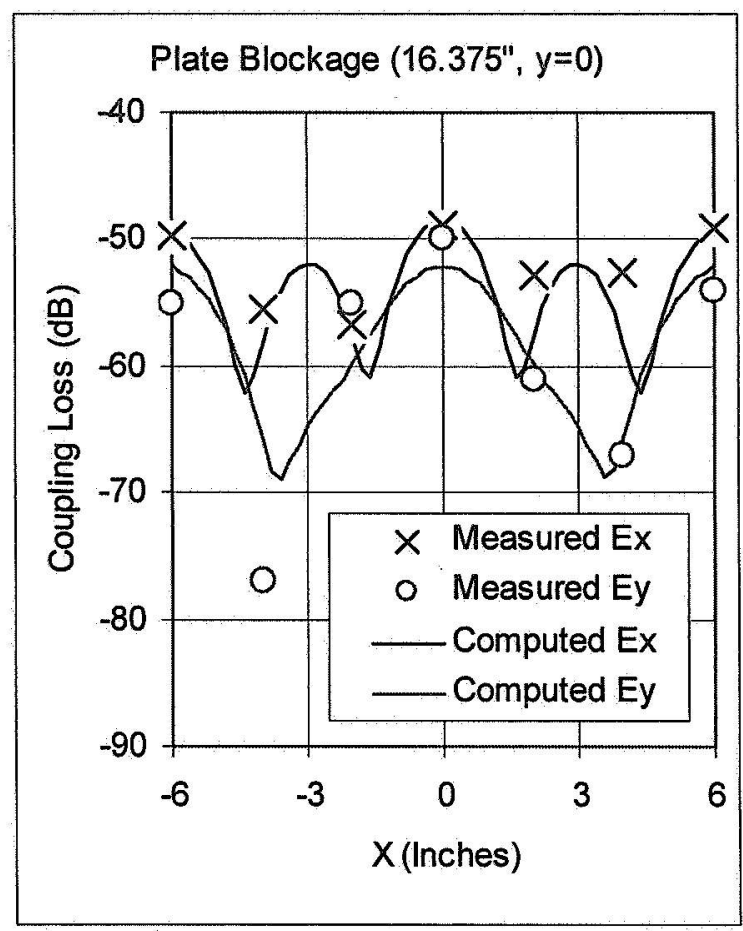

Fig. 4. Measured and computed signal strength along $x$-axis underneath a flat plate.

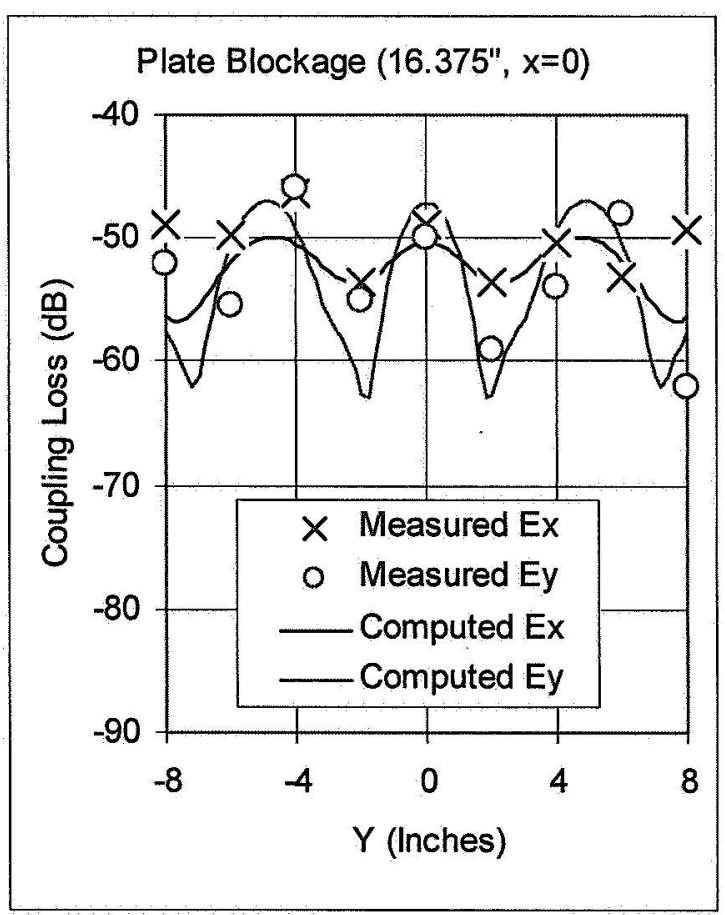

Fig. 5. Measured and computed signal strength along y-axis underneath a flat plate. 


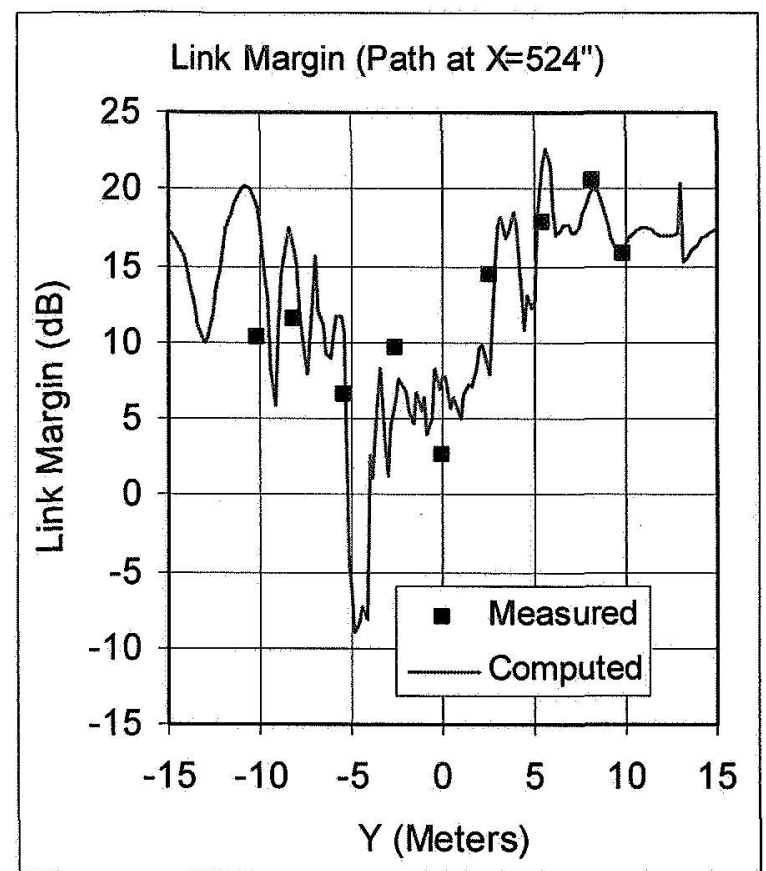

Figure 6. Measured and computed signal strength along an EVA path underneath the Space Shuttle.

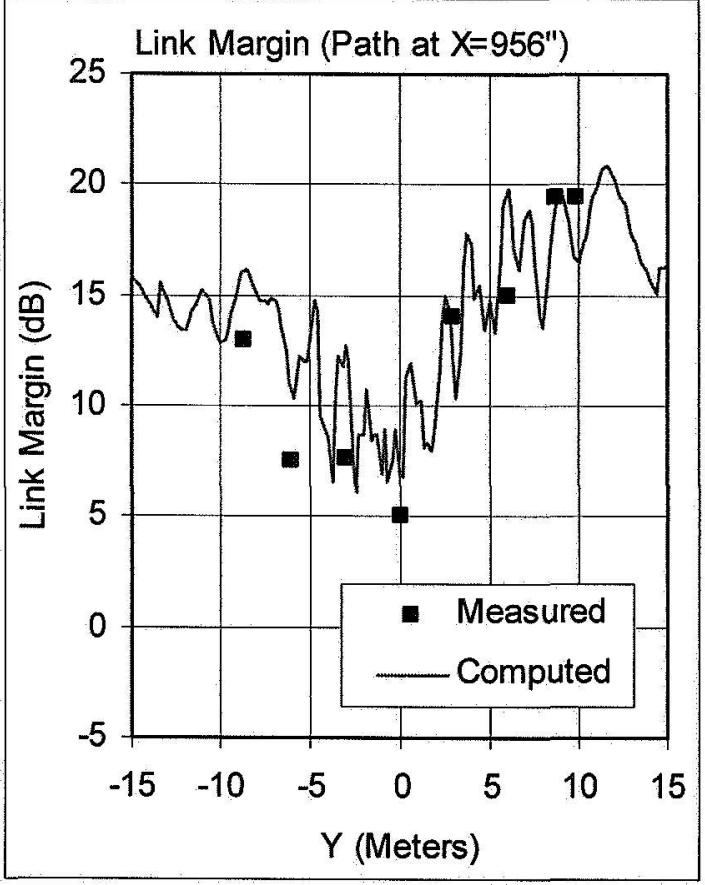

Fig. 7. Measured and computed signal strength along an EVA path underneath the Space Shuttle.

\section{COMMUNICATION PERFORMANCE EVALUATION}

The communications performance is evaluated for EVA communications when astronauts performing vehicle inspection and repair tasks. Data obtained from both the computer simulations and near-field measurements were analyzed.

The Space Shuttle payload bay UHF antenna transmit power is $-3.4 \mathrm{dBW}$. The circuit loss is $-2.1 \mathrm{~dB}$. The UHF antenna signal strengths $(\mathrm{dBV} / \mathrm{m})$ at 2 meters underneath the Space Shuttle Orbiter are shown in Fig. 8. The signal level on the starboard side is higher than the signal level on the port side due to the payload bay UHF antenna location. The data indicates that the lowest signal levels are in the regions near the tail due to the significant wing blockage in those areas. As expected, the signal levels improve with increasing separation distance between the astronauts and the Orbiter underside [8-11].

SSO ant signal $(\mathrm{dBV} / \mathrm{m})$ at $2 \mathrm{~m}$ underneath Space Shuttle

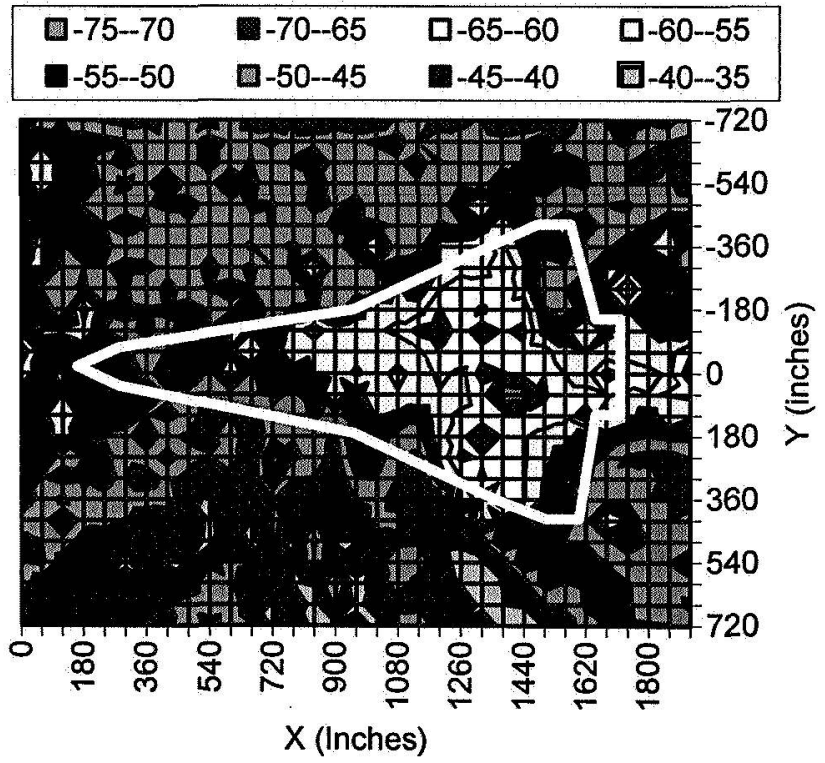

Fig. 8. Space Shuttle UHF antenna signal strengths $(\mathrm{dBV} / \mathrm{m})$ at 2 meters underneath the Space Shuttle Orbiter.

Computer simulations and experimental measurements were also performed for the International Space Station (ISS) UHF Space-to-Space Communications System (SSCS) Lab and P1 truss antennas. The ISS SSCS antennas are available and can be used for the EVA communications when the Space Shuttle is docked to the Space Station. The ISS UHF antenna locations are shown in Figs. 9 and 10.

The Space Station UHF Lab antenna transmit power is -3.9 $\mathrm{dBW}$. The circuit loss is $-4.2 \mathrm{~dB}$. The Space Station UHF Lab antenna signal strengths $(\mathrm{dBV} / \mathrm{m})$ at 2 meters underneath the Space Shuttle are shown in Fig. 11. The signal level on the Shuttle starboard side is much higher than anywhere else due to the ISS Lab antenna location. When the Shuttle is docked to Station, the ISS Lab antenna points generally toward the inside of the open starboard payload bay door. The data indicates that the lowest signal levels are in the regions near the tail due to the significant wing blockage in those areas. The signal level is in general better for the ISS Lab antenna than for the SSO UHF 
antenna due to the ISS Lab antenna favorable location and pointing direction. The ISS UHF Lab antenna is a good candidate as the primary antenna for EVA communications underneath Space Shuttle.

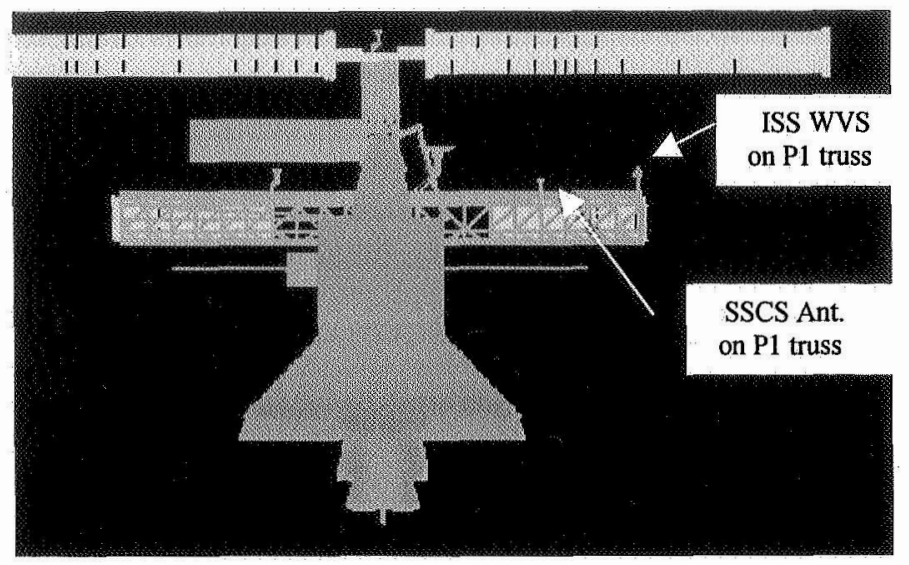

Fig. 9. Front view of Space Station antenna locations.

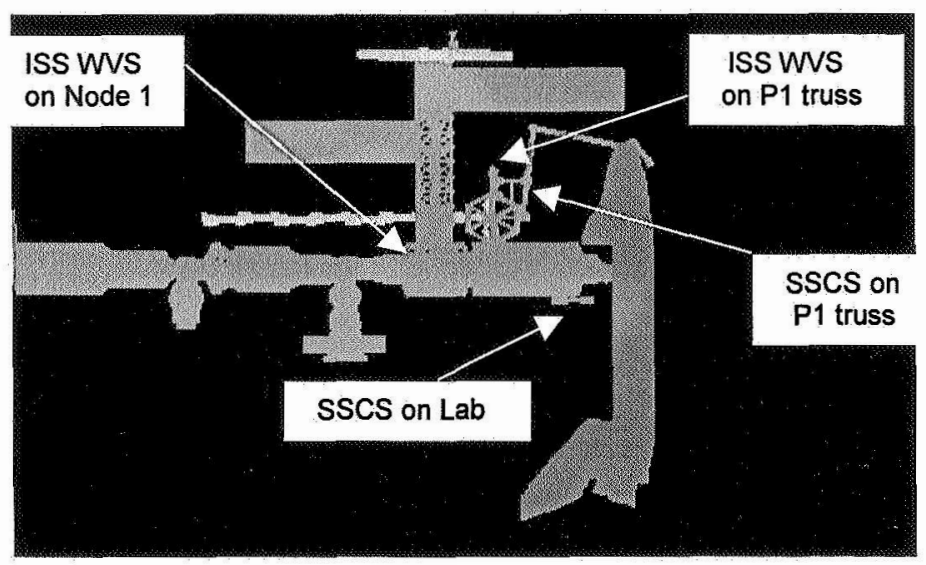

Fig. 10. Side view of Space Station antenna locations.

The Space Station UHF P1 antenna transmit power is -3.9 $\mathrm{dBW}$. The circuit loss is $-8.4 \mathrm{~dB}$. The ISS UHF P1 antenna signal strengths $(\mathrm{dBV} / \mathrm{m})$ at 2 meters underneath the Space Shuttle Orbiter are shown in Fig. 12. The signal level on the Shuttle port side is much higher than anywhere else due to the ISS P1 antenna location. The signal level is in general better for the ISS P1 antenna than for the SSO UHF antenna due to the ISS P1 antenna favorable location and pointing direction. The ISS UHF P1 antenna is a good candidate as the secondary antenna for EVA communications underneath Space Shuttle.

In the near field the electric field generally has three orthogonal components with non-uniform magnitude. In order to obtain the optimum communication performance, an occasional EVA astronaut maneuvering of one or two feet or a slightly different orientation of 10 or 20 degrees may be necessary.
ISS Lab ant signal (dBV/m) at $2 \mathrm{~m}$ underneath Orbiter

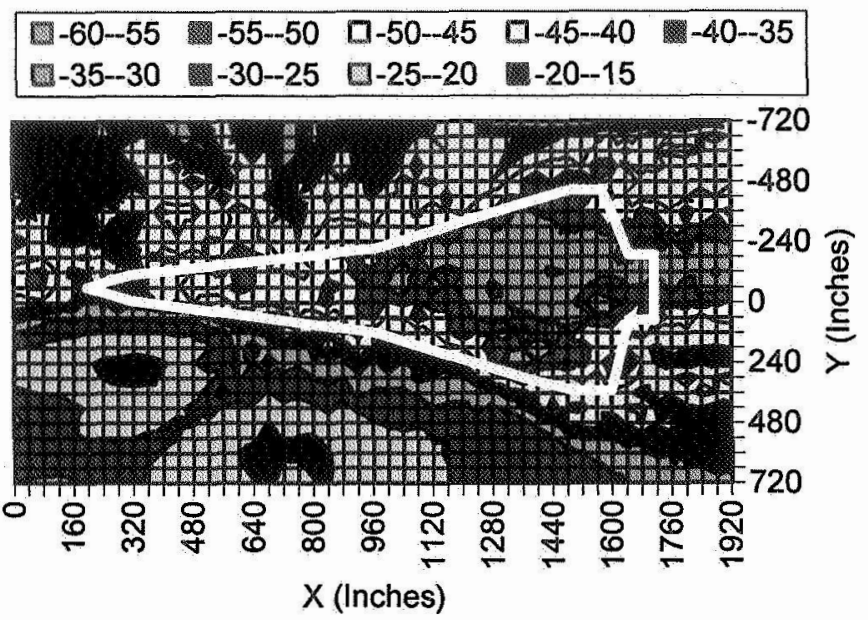

Fig. 11. Space Station Lab antenna signal strengths $(\mathrm{dBV} / \mathrm{m})$ at 2 meters underneath the Space Shuttle.

ISS P1 ant signal (dBV/m) at $2 \mathrm{~m}$ underneath Orbiter

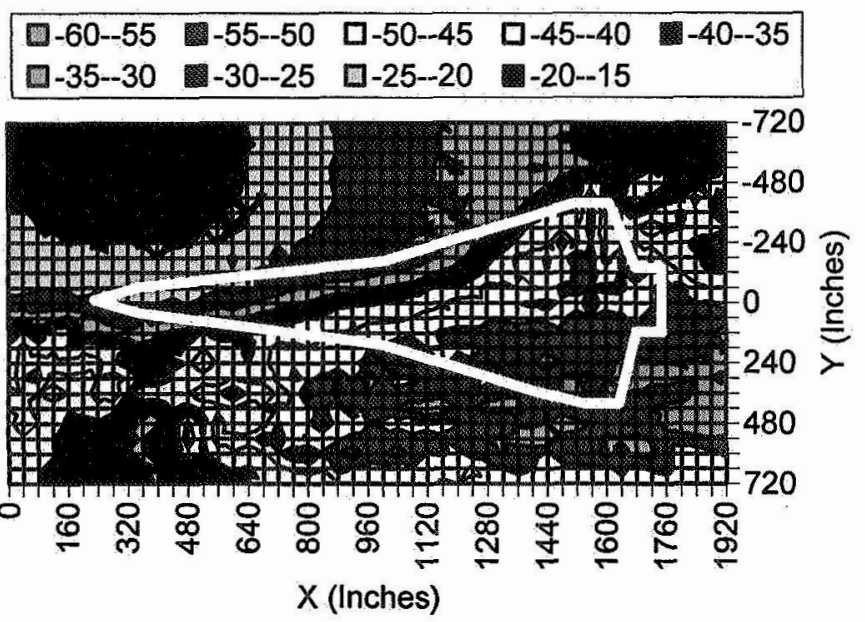

Fig. 12. Space Station P1 antenna signal strengths $(\mathrm{dBV} / \mathrm{m})$ at 2 meters underneath the Space Shuttle.

\section{CONCLUSION}

In this study, the communication performance underneath the Space Shuttle Orbiter using the Space Shuttle and Space Station UHF antennas were analyzed. The shadowing effects from the Space Shuttle vehicle structures blocking the UHF antennas were investigated. The computer simulation tool based on the Geometrical Theory of Diffraction method was used to compute the signal strengths. Based on the results obtained in this study, RF coverage areas for the underside communications were determined.

Good EVA astronaut communication coverage can be achieved by using the Space Station Lab antenna as the primary antenna and switching to the Space Station P1 truss antenna when necessary. The signal levels improve with increasing 
separation distance between the EVA astronauts and the Orbiter underside. The data indicate that the lowest signal levels are in the regions near the tail due to the significant wing blockage in those areas. The signal level is in general better for the Space Station Lab and P1 antennas than for the Space Shuttle payload bay antenna due to the Space Station Lab/P1 antenna favorable location and pointing direction. The Space Station Lab antenna provides better signal levels on the Shuttle port side and the Space Station P1 antenna provides better signal levels on the Shuttle starboard side.

It has been shown that a communication link is possible for many places underneath the Space Shuttle. The areas of poor link are in the deep shadow region and have been identified by this work. An astronaut can possibly improve a poor link condition by a re-orientation. An astronaut relay scenario using a second astronaut to relay messages can also improve the coverage area.

\section{ACKNOWLEDGMENT}

The authors wish to acknowledge the contributions from Wayne Cope of Lockheed Martin for implementation of the near-field measurements. The review and comments by William Culpepper of NASA/JSC are appreciated.

\section{REFERENCES}

[1] R.J. Marhefka, and W.D. Burnside, "Antennas on complex platforms," Proceedings of the IEEE, Volume: 80, Issue: 1, pp. 204-208, Jan. 1992.

[2] W. Burnside, M. Gilreath, R. Marhefka, Yu Chong, "A study of KC-135 aircraft antenna patterns," IEEE Trans. Antennas and Propagation, [legacy, pre - 1988], Volume: 23, Issue: 3, pp. 309-316, May 1975.

[3] R.J. Marhefka, "The other NEC (BSC) an asymptotic complement," Antennas and Propagation Society Symposium, 2004. IEEE, Vol.3, pp. 2911-2914, 20-25 June 2004.

[4] R. J. Marhefka, and J. W. Silvestro, "Near zone - basic scattering code user's manual with space station applications," NASA CR-181944, Dec. 1989.

[5] W.D. Burnside, J.J. Kim, B. Grandchamp, and R.G. Rojas, "Airborne antenna radiation pattern code user's manual." Ohio State University ElectroScience Laboratory Report 712242-14, Dec. 1982

[6] S. Hwu, Y. Loh, L. Catherine C. Sham, and Quin D. Kroll, "International space station Ku-band communications antenna blockage analysis," Journal of spacecraft and Rockets, pp. 232-233, Vol. 41, No. 2, Mar. 2004.

[7] S. Hwu, Y. Loh, L. Johnson, R. Panneton, "Solar array modeling for space station S-band and GPS communications analysis," Journal of spacecraft and Rockets, pp. 132-133, Vol. 40, No. 1, Jan. 2003.

[8] J. A. Dobbins, "Engineering Evaluation of 1/10th Scale Shuttle Payload Bay Antennas in 1/10th Scale Orbiter Mockup," TPS\# 610420032, EV4, NASA Johnson Space Center, July 2004.

[9] S. Hwu, "Space Shuttle Orbiter payload bay UHF SSOR and WVS antenna test data coverage analysis," NASA Johnson Space Center, EV7-04-3058, Sep. 2004.

[10] S. Hwu, J. Boster, and T. Z. Abebe, "Space Underside Communications Performance Evaluation," NASA Johnson Space Center, EV7-05-3104, Dec. 2004.

[11] S. U. Hwu, and T. Z. Abebe, "Space Shuttle Orbiter Payload Bay SSOR and WVS UHF Antenna RF Coverage Requirements Analysis," NASA Johnson Space Center, EV7-05-3103, Nov. 2004. 